\title{
Engaging Cognitive Circuits to Promote Motor Recovery in Degenerative Disorders. Exercise as a Learning Modality
}

\author{
by \\ Michael W. Jakowec', Zhou Wang², Daniel Holschneider' ${ }^{2}$ Jeff Beeler ${ }^{3}$, \\ Giselle M. Petzinger ${ }^{1}$
}

Exercise and physical activity are fundamental components of a lifestyle essential in maintaining a healthy brain. This is primarily due to the fact that the adult brain maintains a high degree of plasticity and activity is essential for homeostasis throughout life. Plasticity is not lost even in the context of a neurodegenerative disorder, but could be maladaptive thus promoting disease onset and progression. A major breakthrough in treating brain disorders such as Parkinson's disease is to drive neuroplasticity in a direction to improve motor and cognitive dysfunction. The purpose of this short review is to present the evidence from our laboratories that supports neuroplasticity as a potential therapeutic target in treating brain disorders. We consider that the enhancement of motor recovery in both animal models of dopamine depletion and in patients with Parkinson's disease is optimized when cognitive circuits are engaged; in other words, the brain is engaged in a learning modality. Therefore, we propose that to be effective in treating Parkinson's disease, physical therapy must employ both skill-based exercise (to drive specific circuits) and aerobic exercise (to drive the expression of molecules required to strengthen synaptic connections) components to select those neuronal circuits, such as the corticostriatal pathway, necessary to restore proper motor and cognitive behaviors. In the wide spectrum of different forms of exercise, learning as the fundamental modality likely links interventions used to treat patients with Parkinson's disease and may be necessary to drive beneficial neuroplasticity resulting in symptomatic improvement and possible disease modification.

Key words: physical activity, Parkinson's disease, cognition, dopamine, neuroplasticity.

\section{Introduction}

It is only recent that two concepts have converged to formulate an improved working model to better understand the potential role of physical therapy in treating neurodegenerative disorders including Parkinson's disease (PD). These include (1) the concept of ongoing neuroplasticity throughout the course of disease and (2) the concept that there is intimate integration of motor behaviors with regions of the brain involved in cognition. These two distinctive concepts are familiar to researchers in the fields of developmental neurobiology and motor control, especially from the perspective of basic neuroscience. We highlight the novel application of these concepts to progressive degenerative brain disorders like PD, and how they support the emergence of a new approach to target neurorehabilitation (Petzinger et al., 2011; Petzinger et al., 2015). Within the last decade, we have begun to appreciate the fact that physical activity and exercise reduces the incidence of PD and may in fact modify disease progression in those already affected (Chen et al., 2005; Thacker et al., 2008). Our short review of this topic outlines a few milestones in the conceptual origins of neuroplasticity throughout history, which emphasize that today's modern ideas are in fact in part centuries old. In addition, we briefly explore

1 - Department of Neurology, University of Southern California, Los Angeles, California, USA.

2 - Department of Psychiatry, University of Southern California, Los Angeles, California, USA.

3 - Department of Psychology, Queens College, City University of New York, New York City, USA. 
some of the research findings that have guided us over the last decade to better understand neuroplasticity and its underlying mechanisms. Finally, through our understanding of cognition and motor behaviors in the human brain, we look at neurodegenerative disorders with a new perspective. From this viewpoint, treatment of these disorders needs to be applied in the context of highly integrated and complex systems, with an understanding that it is not enough to devise pharmacologic strategies to simply restore a missing transmitter. What we learn about the impact of physical activity, exercise and physical therapy in the context of neurological disorders like PD also provides us with valuable insights into mechanisms by which these interventions impact general health of the normal brain from early postnatal development, through adolescence and into all stages of adulthood.

\section{The Concept of Neuroplasticity has its Roots in History}

In the scientific literature over the past 300 years one can find some remarkable examples of insights that parallel recent hypotheses in neuroplasticity. For example, Spurzheim (1815) speculated "Here it may be asked whether the organs of the brain increase by exercise? This may certainly happen in the brain as well as in the muscle. In order to be able to answer this question positively, we ought to observe the same persons when exercised and when not exercised, or at least observe many persons who are, and many others who are not, exercised during all periods of life". The father of evolutionary theory, Charles Darwin, in The Decent of Man (1874), remarked "I have shown that the brains of domestic rabbits are considerably reduced in bulk, in comparison with those of wild hare (...) so that they have exerted their intellect, instincts, senses and voluntary movements but little". Eugenio Tanzi chimed in at the neuronal level when he stated in A Textbook of Mental Diseases (1900), "Perhaps every representation immediately determines functional hypertrophy of the protoplasmic processes and axons concerned; molecular vibrations become more intense and diffuse themselves, momentarily altering the form of the dendrites; and thus, if the conditions are favourable, new expansions and collateral originate and become permanent" (1880) (Tanzi, 1909). His student,
Ernesto Lugaro, writing in Modern Problems in Psychiatry (1906) introduces the term neuroplasticity into the neurosciences complementing Williams James' introduction of the term into the human psychology nomenclature as he outlined in The Principles of Psychology in 1890.

During this same time period, Santiago Ramon y Cajal writing in Les Nouvelle Idées sur la Structure du Système Nerveux chez l'Homme et chez les Vertébrés (1895), unaware of neurogenesis in the adult brain, put forth the speculation that "One might suppose that cerebral exercise, since it cannot produce new cells, carries further than usual the development of protoplasmic expansions and neural collateral forcing the establishment of new and more extended intercortical connections". It is interesting to note that Cajal further explored the concept of environmental impact on brain function as a counterpart to genetic determinism when he stated in Degeneration and Regeneration of the Nervous System (1933) "Morphology of the nerve cell does not obey an immanent and fatal tendency, maintained by hereditary, as certain authors have defended, but it depends entirely on physical and chemical circumstances present in the environment". Based on his observations of the dynamics of dendritic spines, he postulated that dendrites were transient and crucial to normal brain function and that neurons could both expand and retract during learning and memory (Croonian Lecture, 1894). In contrast Ramon y Cajal is often quoted as supporting the inflexibility of the adult brain when he stated in Estudios Sobre la Degeneración y Regeneración del Sistema Nerviosa (1913-1914), "Nerve paths are somewhat fixed, ended, immutable. Everything may die, nothing may regenerate". The notable quotes at different phases of his career contrasting hypotheses underscoring the development of his thinking.

After the period involving such eminent scientists as Charles Sherrington around 1920, the concept of neuroplasticity in the adult brain lay dormant and certainly out of fashion for several decades. A major re-emergence in neuroplasticity was led by two influential publications in the late 1940's. In 1948, in Conditioned Reflexes and Neuron Organization Jerzy Konorski predicted that associative learning may be due to long-term 
changes in neuronal excitability, defining such changes as "plastic". This is emphasized in his statement, "The plastic changes would be related to the formation and multiplication of new synaptic junctions between the axon terminals of one nerve cell and the soma (i.e. the body and the dendrites) of the other". At almost the same time, Donald Hebb stated in his 1949 publication, The Organization of Behavior: A Neuropsychological Theory, "When an axon of cell A is near enough to excite a cell $\mathrm{B}$ and repeatedly and persistently takes part in firing it, some growth process or metabolic change takes place in one or both cells such that A's efficacy, as one of the cells firing B, is increased". This quotation has become the battle cry of the "use it or lose it" phenomenon as well as the coined phrase "cells that fire together, wire together" (Shatz, 1992).

That neuroplasticity is fundamental not only in establishing brain connectivity, but also in its life-long maintenance was proposed by the physiologist Walter Cannon in The Wisdom of the Body (1932). "By an apparent contradiction, it (the brain) maintains its stability only if it is excitable and capable of modifying itself according to external stimuli, and adjusting its response to the stimulation. In a sense it is stable because it is modifiable - the slight instability is the necessary condition for the true stability of the organism". This proposal is a conceptual model of neuronal homeostasis that suggests that the continuous dynamic nature of the mammalian nervous system is necessary for maintaining optimal function throughout life.

Research by Rosenzweig, Krech, Bennett and Diamond in 1962 ushered in a new era in neuroscience by demonstrating that "manipulating the environment after weaning can alter the weight of the cerebral cortex and activity of the brain" (Rosenzweig et al., 1962). The underlying morphological changes in dendritic structure at the resolution of electron microscopy reported by Greenough and colleagues gave further weight to the influence of environment on neuronal structure and connectivity and even delineated differential effects of skill-based and aerobic forms of physical activity (Black et al., 1990). Despite significant findings indicating changes in rodent brain weight and cortical thickness due to interactions with the environment, there was little evidence and in fact active resistance towards the concept that such phenomena occur in higher primates including humans. We are now at the stage where hundreds of publications, many utilizing a wide spectrum of neuroimaging techniques, have shown changes in activation and circuitry with physical activity in human subjects (Caproni et al., 2013; Hamaide et al., 2016). Several reviews articulate in greater detail than our present brief synopsis, the history of the scientific concept of brain plasticity and we refer interested students of the neurosciences to these, as well as the tests of aforementioned authors (Berlucchi, 2002; Berlucchi and Buchtel, 2009; Cooper, 2005; Stahnisch, 2003).

\section{Defining Neuroplasticity}

Neuroplasticity is a term to describe the brain's ability to reorganize itself by forming new neural connections throughout all stages of life from the early postnatal period, through puberty, adolescence and into early and late adulthood. While the most robust period of neuroplasticity as influenced by activity and the environment occurs during a very narrow developmental window called the critical period, it is now recognized that this window may never be fully closed, and in fact, we are beginning to identify mechanisms by which we can re-open this developmental window in the adult when the critical period is long passed (Bavelier et al., 2010). Neuroplasticity allows neurons and their circuits within the brain to compensate for injury and disease and to adjust their activities in response to changes in their environment. We can describe neuroplasticity at many different stages or levels within the brain most often based on the technical approaches of specific experimental measures. For example, electrophysiologists consider neuroplasticity in the context of changes in long-term potentiation (LTP) or long-term depression (LTD) manifesting as altered excitation properties of circuits due to changes in receptor distribution at the synapses. On the other hand, researchers in the field of biokinesiology or physical therapy would consider neuroplasticity in terms of changes in behavior in response to training or exercise. These two divergent ends of a spectrum can be linked through neuro-imaging in human subjects or through molecular analysis in animal models where regional anatomical changes in activation, circuitry and connectivity can be documented. 


\section{Exercise Enhances Recovery of Motor Behavior in Parkinsonian Rodent Models}

In the classical pathophysiological model of $\mathrm{PD}$, the loss of dopamine in the dorsal lateral striatum leads to imbalance of the dopamine D1 receptor containing the "direct" pathway and the dopamine D2 receptor "indirect" pathway. This then leads to increased corticostriatal glutamatergic synaptic drive and hyperexcitability in the indirect pathway. One means to normalize this aberrant circuit may be through restoration of dopamine neurotransmission, including elevating the expression of the dopamine D2 receptor. Our findings in animal models of dopamine depletion seem to support this approach. For example, according to studies in the 1-methyl-4-phenyl-1,2,3,6tetrahydropyridine (MPTP) mouse model of PD, intensive daily treadmill exercise leads to improved motor function and increased dopamine neurotransmission compared to the non-exercised MPTP mice. Exercised mice showed: (i) increased evoked dopamine release, (ii) increased extracellular dopamine though down-regulation of the dopamine transporter (DAT) expression, and (iii) decreased clearance as measured by fast-scan cyclic voltammetry within the dorsal striatum (Fisher et al., 2004; Petzinger et al., 2007). Not only are there changes in the expression of the dopamine D2 receptor, but these changes influence and restore motor learning, a behavior dependent on the dopamine D2 receptor, some of which may be regulated through glutamate neurotransmission originating from the corticostriatal pathway (Kintz et al., 2013; VanLeeuwen et al., 2010). Thus, exerciserelated changes in dopamine availability through altered neurotransmission may act in part to promote mechanisms critical for motor learning and that are important for restoring motor behavior in PD. Using positron emission tomography imaging with $\left[{ }^{18} \mathrm{~F}\right]$-fallypride, a ligand with high specificity for the dopamine D2 receptor, this effect of exercise in MPTP-lesioned mice was also observed through increased dopamine D2 receptor binding (Vuckovic et al., 2010). These reports are consistent with studies that demonstrate an exercise-induced increase of dopamine D2 receptor mRNA, protein and binding in the striatum of healthy non-dopamine depleted rodents (Foley and Fleshner, 2008; Gilliam et al., 1984; MacRae et al., 1987). Translating these findings from the MPTPdepleted rodent model to clinical studies in patients with $\mathrm{PD}$, an exercise-induced increase in dopamine D2 receptor expression was also observed in individuals who were newly diagnosed and underwent treadmill exercise (Fisher et al., 2013). After an 8 week regimen of intensive treadmill training, PET-imaging demonstrated an 80 to $90 \%$ increase in binding of $\left[{ }^{18} \mathrm{~F}\right]$-fallypride within the dorsal caudate nucleus compared to pre-exercise baseline values. There was no change in receptor availability in PD subjects in the absence of exercise and also no change in normal subjects with exercise, thus supporting a disease specific effect of the intervention. While the relationship between exercise-induced motor benefits in PD and increased dopamine D2 receptor expression in humans is unknown, studies in healthy animals suggest that striatal dopamine D2 receptor function and its role in the establishment and maintenance of motor skill learning may underlie this benefit (Beeler et al., 2012; Yim et al., 2009). For example, electrophysiological studies within the striatum of animals, in conjunction with a pharmacologically specific blockade of dopamine D2 receptors, have shown that dopamine D2 receptor antagonism in either the early or late phases of motor skill learning leads to impairment in glutamatergic-dependent synaptic potentiation and motor learning (Yim et al., 2009). These studies also demonstrate a dopamine D2 receptor related synaptic plasticity that is responsible for motor learning and which is localized in the dorsal striatum. Further support for the role of the dopamine D2 receptor and motor learning comes from studies in rodents by Beeler and colleagues (Beeler et al., 2010; Beeler et al., 2012). These studies demonstrate that the dopamine D2 receptor is also important in the maintenance of learned motor behaviors since pharmacological blockade of the dopamine D2 receptor, and not the dopamine D1 receptor, leads to loss of a learned motor skill. In addition to its role in motor performance, studies in animals suggest that an exercise-induced increase in dorsal striatal dopamine D2 receptor expression may also contribute to the reported exercise related 
improvements in executive function, including behavioral flexibility (Eddy et al., 2014). Specifically, studies have shown that 18 days of exercise can improve discrimination testing in a set-shifting, cross-maze task in healthy rodents. This exercise benefit was reversed through selective pharmacological blockade of the dopamine D2 receptor. Taken together animal studies support that an exercise induced increase in dopamine availability along with increased dopamine D2 receptor expression in the dorsal striatum and its related cortical circuitry contribute to exercise related effects in neuroplasticity and behavioral benefits in PD. Future studies in humans are clearly needed to confirm this relationship.

\section{Blood Flow Studies in Parkinsonian Rodents implicate a Role for Cognition}

Historically, studies on neuroplasticity in models of dopamine depletion have focused on the striatum (caudate-putamen), the primary site of affected motor circuits in PD. Recent work exploring region specific changes have identified pathways outside the basal ganglia that influence the recovery of motor circuits. Blood flow studies in parkinsonian rodents engaged in exercise indicate that not only are there differences between normal and dopamine-depleted subjects, but also differences based on the type of exercise the animal is engaged in (Wang et al., 2013; Yang et al., 2007). While we do know that cognitive and motor circuits are closely integrated especially through the corticostriatal pathway, these findings have important implications in supporting our hypothesis that the type of exercise is critical for enhancing motor behavior recovery. From a categorical point of view, the exercise type can be simply delineated into either a form that is predominantly aerobic or a form that is predominantly skill-based in nature. Aerobic exercise can be defined as a system of conditioning aimed at enhancing circulatory and respiratory efficiency that improves the body's use of oxygen through vigorous, sustained exercise such as running, swimming or cycling. This is in contrast to skilled-based exercise, which can be defined as a form of goal-oriented movement in which temporal and spatial accuracy is important for achieving pre-determined objectives. Studies in rodents have shown differences in the impact of these two forms of exercise in measures of brain morphology. For example, rats that underwent unskilled and repetitive exercise (aerobic exercise) had an increase in the density of blood capillaries in the brain's motor regions, without an increase in synaptic numbers (as measured by dendritic spine density) (Black et al., 1990; Garcia et al., 2012). This is in contrast to rats that learned new motor skills (skilled-based exercise) and had a greater number of synapses per neuron, without an increase in the density of blood capillaries. Extending these observations, recent work by our laboratory indicates that skill-based compared to non-skilled aerobic training differentially affects functional activation of the medial prefrontal cortex (mPFC) in parkinsonian rats during walking (Wang et al., 2015). Rats with bilateral, striatal 6-OHDA lesions were exposed to forced exercise for 4 weeks, either on a simple running wheel, considered a form of non-skilled aerobic exercise, or on a complex wheel with irregularly spaced rungs, a form of skill-based exercise in which the animal must be cognitively engaged with its task. Cerebral perfusion was mapped during treadmill walking or at rest using $\left[{ }^{14} \mathrm{C}\right]-$ iodoantipyrine autoradiography one week after the completion of exercise. Skill-based exercise compared to aerobic exercise resulted in greater increases in the regional cerebral blood flow $(\mathrm{rCBF})$ during walking and at rest in the prefrontal cortex (prelimbic area). Seed correlation analysis during locomotor walking revealed that skill-based exercise compared to aerobic exercise resulted in a much broader functional connectivity of the prefrontal cortex with the striatum providing evidence of frontal-striatal neuroplasticity in these circuits. Such findings suggest that skill-based exercise requires greater effort in cortical processing, motor control and flexibility compared to that required for aerobic exercise. The PFC controls these cognitive behavioral features. Therefore, in its application to patients with $\mathrm{PD}$ and motor rehabilitation programs, subjects should engage in a relatively high cognitive demand, such that by forcing patients to practice task-switching over a sufficient number of practice trials, they might be able to overcome their inability to generalize learned actions to different environmental contexts. While dopaminergic dysfunction in the PFC is an early feature of PD and has been linked 
to dopaminergic loss in the caudate and substantia nigra (Berti et al., 2010; Polito et al., 2012), its role in shaping cognitive deficits and responding to an exercise intervention remains to be determined.

\section{Thinking About Different Forms of Exercise}

Studies from animal models subjected to different forms of exercise have enticed us to reevaluate the different forms of exercise, especially those utilized in physical therapy for patients with PD. As already stated, we can begin to define the two poles of the exercise spectrum from skillbased exercise to aerobic exercise. Skill-based exercise can be defined as a form of goal-oriented movement in which temporal and/or spatial accuracy is important for achieving predetermined objectives such as movements in yoga, Tai Chi or amplitude training in patients with PD. On the other hand, aerobic exercise can be defined as a system of conditioning aimed at enhancing circulatory and respiratory efficiency that improves the body's use of oxygen through vigorous sustained exercise such that observed in running, swimming, cycling. Of course almost all forms of human physical activity utilize some fraction of either skill-based or aerobic exercise and it is impossible to have any form of physical activity that is exclusively one and not the other. However, any given physical exercise regimen can certainly emphasize skill-based activity while another can emphasize aerobic activity.

Studies are beginning to define with greater precision the contribution of skill-based versus aerobic forms of exercise. However, it is hypothesized that for the establishment and maintenance of specific motor circuits and their respective behaviors, both are required. One can begin to develop a model of how this may occur. For example, skill-based exercise such as learning to play tennis or ice skate requires intensive concentration (cognitive engagement) where the subject focuses on the physical activity, receives feedback (from a coach for example, or falling), and assesses this success or failure to readjust, eventually leading to meaningful behavior. Distraction can impede learning of new motor skills. Aerobic exercise or the aerobic component of the physical activity plays a critical role in making the neuronal environment conducive for the selection and optimization of necessary neuronal circuits. For example, there is evidence that neurotrophic factors such as the brainderived neurotrophic factor (BDNF) or nerve growth factor (NGF) can lower the threshold by which physiological changes can take place to strengthen synaptic connectivity, and therefore allow specific neuronal pathways to be reenforced for long-term utilization (Adlard and Cotman, 2004; Berchtold et al., 2005). Once these circuits are established, practice may be necessary to maintain their integrity at a high level and to avoid the "use-it-or-lose-it" phenomena that could result. In other words, these circuits are not simply fixed or hardwired in the brain, but are subject to mechanisms such as homeostatic neuroplasticity, where their continuous activity maintains their desired function.

\section{Linking Cognition and Motor Behavior}

Cognition is the set of all mental abilities and processes related to knowledge including attention, memory and working memory, executive function, judgment and evaluation, reasoning and computation, problem solving and decision making, comprehension and production of language. Human cognition is conscious and unconscious, concrete or abstract, as well as intuitive (for example knowledge of a language) and conceptual (for example a model of a language). Cognitive processes use existing knowledge and generate new knowledge. Consequently, this description tends to apply to processes such as memory, association, concept formation, pattern recognition, language, attention, perception, action, problem solving and mental imagery. Motor behavior is defined as the study of how motor skills are learned, controlled and developed to assist people as they practice and experience physical activity. It is only recently that neuroscience as a field is beginning to appreciate the fact that these similarities in complex features of cognition are also shared with motor behavior. This leads us to better understand that cognition and motor behavior are not mutually exclusive systems, but are closely integrated to control movement and to make basic motor behaviors automatic. It is the loss of automaticity that is highly prevalent in $\mathrm{PD}$, such that patients must think about their every movement and undergo a significant increase in 
the cognitive load that in itself is aberrant and leads to maladaptive neuroplasticity in corticostriatal circuits (Beeler et al., 2013). A goal of physical therapy is to restore automaticity.

\section{The Value of Skilled-exercise in Conjunction with Aerobic Exercise to Promote Motor Recovery}

What are the factors that link the central nervous system (CNS) with the body including its muscle, adipose tissue and peripheral organs? How do peripheral factors participate in reinforcing and consolidating cortico-motor circuits? A wide range of small molecules have been identified that may link the CNS and periphery signaling. For example, neurotrophic factors are activated with exercise in a diverse range of regions throughout the brain. The brainderived neurotrophic factor (BDNF) has been a popular subject since its identification as one factor elevated in expression with exercise (Cotman et al., 2007). Neurons elevated in activity are responsive to the BDNF due to increased expression of its receptor TrkB. BDNF-TrkB interactions can lower the threshold to the induction of long-term potentiation (LTP), thus leading to selective strengthening of activated circuits. While, serum levels of BDNF are elevated with exercise, it is not yet clear as to the mechanisms by which it signals across the blood brain barrier (BBB), and if there is a direct link between peripheral and central sources of the BDNF affecting the brain (Bowling et al., 2016). The metabolism of glucose and fat in the periphery elevates expression of such factors as insulin, ghrelin, irisin and other small molecules that are able to cross the blood brain barrier (BBB) or interact with receptors at the BBB. For example, the brain expresses insulin-like growth factor receptors (IGF-1R) and interactions with its various ligands signal specific metabolic and morphological changes in activated neurons (Dyer et al., 2016).

The elevated activity of neurons within corticostriatal circuits during exercise leads to an increase in metabolic demand, primarily through reduced levels of cellular oxygen. Such a hypoxic metabolic state leads to the activation of factors including hypoxia-inducible factor 1 alpha (HIF$1 \alpha)$, a transcription factor that targets the expression of hundreds of genes involved in a wide spectrum of cellular functions ranging from the metabolism, mitochondria integrity, signaling cascades such as those involving nitric oxide synthase (NOS) and glutamate, synaptogenesis, and morphology (Correia and Moreira, 2010). The result of HIF-1 $\alpha$ activation not only leads to the restoration of normal neuronal function, but also the selection of specifically activated neuronal circuits that underlie the behavior such as those engaged in the acquisition of new motor skills. Thus, the orchestration of intrinsic changes at the level of the neuron and its connections, as well as the influence of peripheral factors through the vasculature, together can begin to formulate a potential working model. Skill-based exercise and its activation of corticostriatal circuits, in conjunction with aerobic exercise and its activation of peripheral factors may help strengthen the functional and structural connectivity of shared neuronal pathways. How these mechanisms differ in the normal adult brain in comparison with the dopamine-depleted brain of PD patients is an active area of research in our laboratories and those of others.

\section{Does Exercise Enhance Neurogenesis?}

The adult mammalian brain maintains a high degree of neurogenesis throughout life. This was first demonstrated in rodents (Altman and Das, 1965), then in nonhuman primates (Kornack and Rakic, 1999) and eventually in humans (Eriksson et al., 1998). While neurogenesis occurs throughout adulthood, it is restricted to a small number of anatomical sites, including the subventricular zone (SVZ), the granule cell layer of the hippocampus, and olfactory (Cameron and Glover, 2015). In normal rodents, both exercise and environmental enrichment increase the rate of birth of newborn cells, their survival, and elevate the proportion of cells that differentiate into neurons, as well as the proportion that successfully incorporates into neuronal circuits affecting behaviors such as learning and memory (van Praag et al., 1999, 2005). Again studies show that increased neurogenesis occurs in those regions of the brain where the emergence of newborn cells predominates ordinarily.

Regions outside these anatomical sites including the striatum do not show enhanced neurogenesis with increased physical activity. 

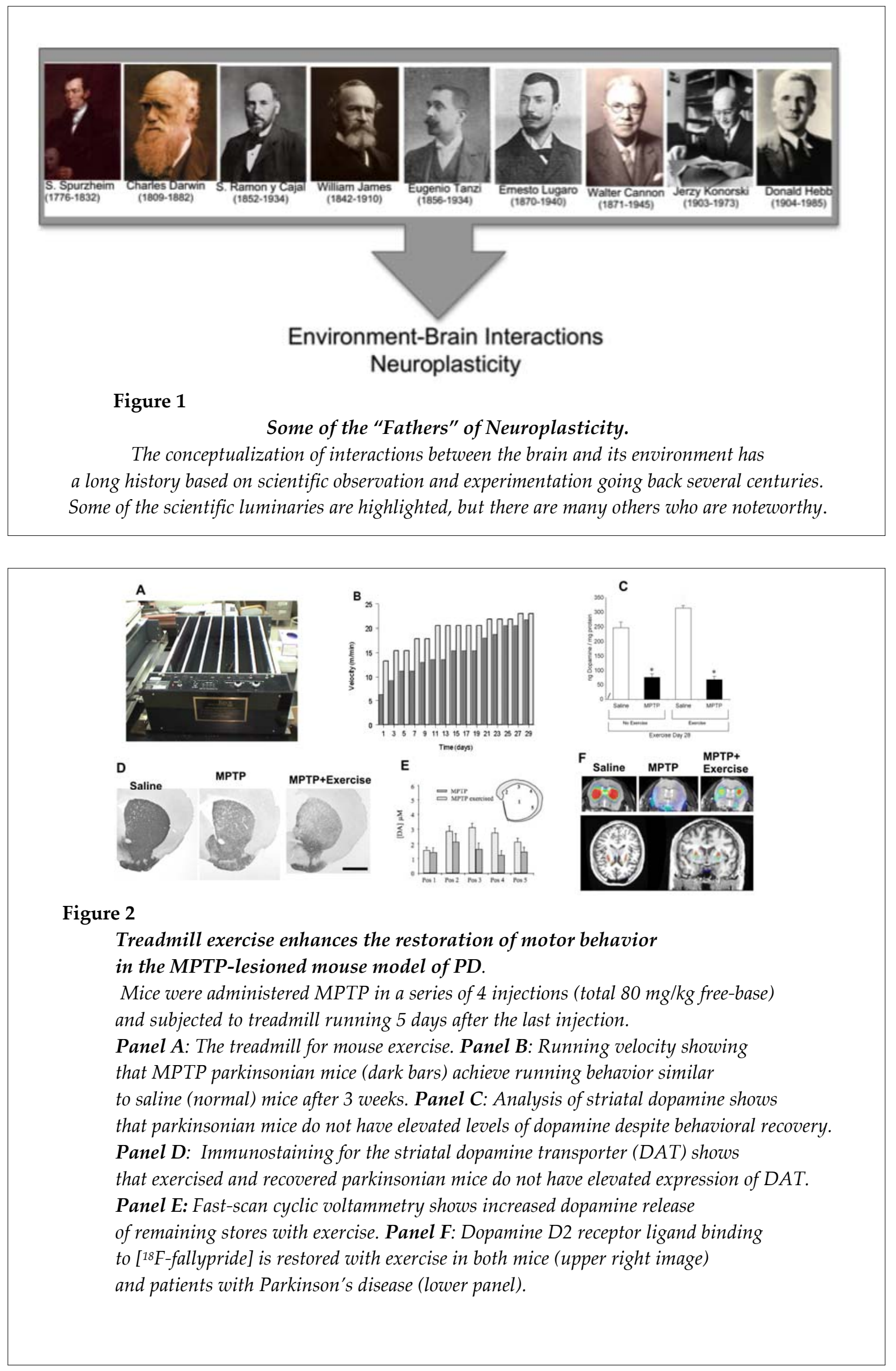


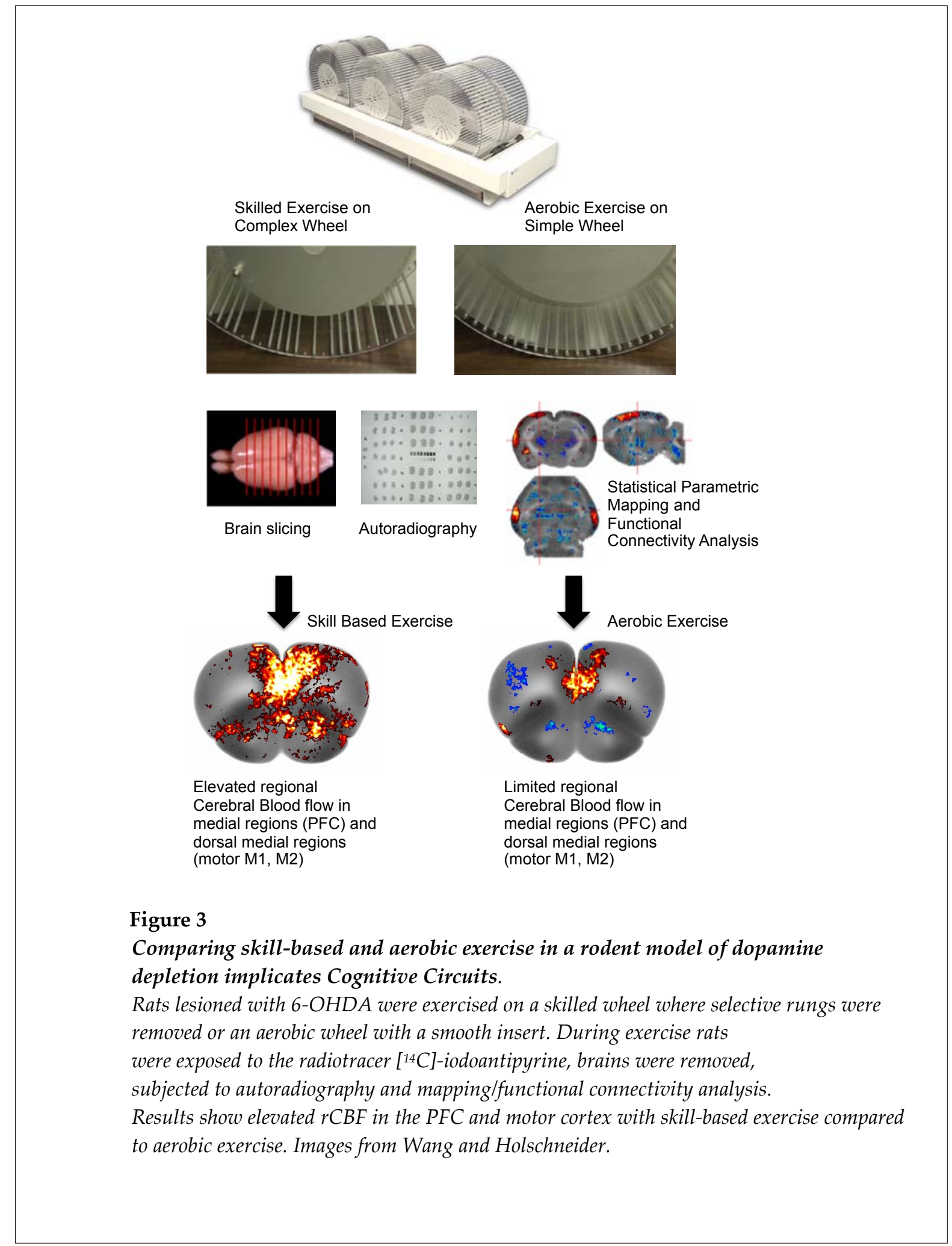

Currently there are few reports of the effects of exercise in animal models of dopamine depletion with respect to enhanced neurogenesis. Some studies have shown reduced gliosis in these models, but similar to studies in normal animals, enhanced neurogenesis is limited to those regions already privileged with cell birth. Interestingly, exercise enhances the survival and integration of transplanted cells in animal models of PD. This is indicative of the importance of experience in 
influencing integration into circuits that are potentially meaningful for functional motor behavior (Dobrossy and Dunnett, 2006; Dobrossy and Nikkhah, 2012).

\section{Additional Major Gaps in Knowledge to Address}

There remain many questions and opportunities in the field of neuroplasticity, especially in the application of studies from animal models and how they can be applied to better understand the effects of physical activity in patients with PD. Below we highlight a few examples.

\section{Motor Imagery}

It is known that the visualization of a motor skill prior to or during a physical activity influences the success of the execution of a task. For example, prior to the start of the giant slalom race, a skier takes several moments to visualize each turn or just before speeding down the runway a gymnast sees in their mind the leaps and twists they will execute over the pommel horse. With greater technological advancements in virtual reality (VR) and the availability of more inexpensive and economical equipment, we are beginning to explore the role of VR in motor learning. In the context of $\mathrm{PD}$, treadmill training in conjunction with VR has begun to reveal how these two approaches can synergistically be applied to promote improvement in the gait and balance symptoms (Mirelman et al., 2011, 2013). As we have hypothesized in our animal models, we believe that the introduction of VR to the treadmill intervention adds a layer of skill not necessarily achieved with a treadmill alone. Thus, cortical inputs to the motor circuitry are enhanced leading to improved behavioral outcome measures. Just as a treadmill alone may not be optimal in maximizing benefits of an intervention, cognitive training alone may also not be sufficient if one wishes to target motor circuits. The result is the need to design interventions that have a high degree of skill as a major component of learning and a high aerobic demand. Together these two factors work in unison to establish and strengthen motor circuits. It is interesting to note that these components are intimately involved in motor learning as that which occurs in early postnatal life, which we sometimes fail to recognize in our understanding of the rationale for physical therapy.

Impact of Exercise Throughout Life, Especially in Adolescence

The vast majority of our focus on the utility of physical therapy is often in the context of injury or brain disorders. We appreciate the importance of physical activity, exercise and playfulness during the early developmental stages of life when complex motor skills emerge and become established. Prime examples include children learning to ride a bicycle, ice skate and other sports demanding high levels of motor mastery and skill. Even though the majority of motor behavior is consolidated in the first decade of life, very little is known about the mechanisms involved in sustaining and developing such skills in the adolescent brain, the period from puberty to adulthood, typically 13 to 25 years of age. We do know that this period in development is one in which the mechanisms of neuroplasticity are active and impacted by activity differently than similar experiences later in adulthood. For example, we do know that the adolescent brain is highly susceptible to drug and alcohol addiction during this stage of development. Experiences including trauma and stress, which underlie posttraumatic stress disorder (PTSD), notably potent in the adolescent brain where brain circuits involved in reward and emotion are still in development.

A number of studies are beginning to reveal how the young brain deals with early stages of disease. For example, in Huntington's disease (HD) where genetic screening for the CAG expansion can identify those individuals at risk, it has been shown that significant brain atrophy occurs early, even decades before the onset of typical HD motor symptomology. Studies including TRACK-HD and PREDICT-HD are providing valuable insights into the life-long course of HD (Biglan et al., 2013; Tabrizi et al., 2013). Despite the fact that the CAG expansion of the huntingtin gene displays complete penetrance, there is a high degree of variability for the age of onset implicating the impact of environmental factors, including lifestyle on the emergence of disease (van Dellen et al., 2005). Unfortunately, very little is currently known regarding the potential impact of physical activity on disease symptom severity and other factors that may modify disease progression. These studies in HD 
could shed light on how the human brain can compensate over a period of many decades before the onset of typical symptomology while battling a neurodegenerative disorder. Currently, we do not have reliable biomarkers for PD and $\mathrm{AD}$ and cannot determine when brain changes occur prior to the emergence of clinical motor and cognitive symptoms. There are researchers who speculate that PD and AD may be similar to HD in that they begin several decades before the onset of symptoms, and thus any intervention to change the course of disease may also have to be applied much earlier in life to influence onset or to optimize any beneficial impact (Meissner, 2012; Selkoe, 2012; Tolosa and Pont-Sunyer, 2011).

\section{Exercise and the Immune System}

Both the innate and acquired components of the immune system are essential for general health in combating disease and infection. The immune system plays a critical role in the CNS in both early life during development, and also during brain injury or disease. In terms of neurodegenerative disorders, activation of components of the immune system, including gliosis and astrocytosis, are seen in $\mathrm{PD}, \mathrm{AD}$ and their animal models (Kannarkat et al., 2013). Blocking or pharmacologically targeting aberrant immune components may be protective and serve as a potential therapeutic target. While very little is known about how exercise changes the immune response in disease, there is some evidence that it may alter microglia activation, astrocytic response or influence the expression of specific pro- and anti-inflammatory factors (Archer et al., 2011; Svensson et al., 2015). Exercise may promote the infiltration of peripheral macrophages to cross the BBB or enhance the conversion of activated microglia from the M1 (pro-inflammatory) to M2 (pro-repair) subtypes for example (Tang and Le, 2016). The fact that microglia play an active role in modulating the number of dendritic spines, a process termed synaptic pruning, directly implicates their potential role in synaptogenesis and neuroplasticity (Schafer et al., 2012). The key to future studies will be to determine if the advantageous aspects of the immune system can be harnessed to promote disease modification possibly through exercise.

\section{The Microbiota and Exercise}

Over the last few years the microbiota (and its collection of genes termed the microbiome) has received much attention in both health and disease. The microbiota primarily consists of the population of microbes found in the gut and on the skin and is responsible not only for the metabolism of a range of factors, but also for maintaining homeostasis (El Aidy et al., 2016; Mayer et al., 2015). We are now beginning to appreciate that the microbiota changes with aging and disease. For example, in several diseases there are significant changes in the content of the microbiota in terms of composition as based on functional and sequencing studies (Evrensel and Ceylan, 2015; Nemani et al., 2014; Scheperjans et al., 2015). In PD, dopamine depletion is not only evident in the CNS, but is also detected in the PNS that innervates the gut. Dopamine depletion may thus underlie changes in gut motility, with constipation as an early symptom in many patients, and with these changes the composition of the microbiota can change with time. The link of the microbiota with the CNS has yet to be elucidated in terms of the mechanism, but it is believed that the two may be interdependent with some degree of a cause and effect relationship. Diet and exercise can change the composition of the microbiota (Kang et al., 2014; Ray, 2014). It would be interesting to know if exercise can alter the microbiota in both patients with PD and in animal models of dopamine depletion. If there are changes, it may implicate another important link bridging the peripheral system and the CNS through exercise.

\section{Can the Effects of Exercise in PD Transfer to Other Neurological Disorders?}

Our studies have shown that exercise, including forms that utilize some degree of cognitive engagement leads to the restoration of motor behavior in animal models of dopamine depletion. The value of this finding would be greatly enhanced if we could transfer such effects to other neurological disorders including models of neurodegenerative disease. To investigate the potential impact of exercise in other models we have conducted studies in a mouse model of Huntington's disease (HD), specifically the knock in (KI) mouse model (termed the CAG140 KI mouse) that has an expansion of 140 CAG repeats in the huntingtin mouse ortholog (Menalled et al., 2003). This model begins to develop HD-like 
motor symptoms after 1 year of life providing a valuable template to test the impact of an early lifelong intervention like exercise. In our laboratory, $\mathrm{CAG}_{140} \mathrm{KI}$ mice and wild-type controls started treadmill running three times per week for one hour when weaned at 1 month of age and this exercise regimen continued for 12 months. Sedentary littermates served as controls. Our findings indicated that HD mice undergoing exercise showed an attenuation of early HD symptoms (anxiety and depression) behaviors that emerged in this model at about 6 months of age. At 12 to 14 months of age there also was an attenuation of early HD-like motor deficits in the gait and balance. Analysis of changes in molecular markers of neuroplasticity and pathology indicated that exercise leads to a reversal in the decline in dopamine D2 receptor expression that occurs with disease, as well as the delay in the accumulation of huntingtin (HTT) protein aggregates, the pathological hallmark of HD in this model. These findings are included in manuscripts currently under review.

While much of our research is carried out in the context of neurodegenerative brain disorders, the effects of exercise have been explored in other neurological disorders that may not have synaptic and neuronal degeneration as the primary etiology. For example, others and we have examined the effects of exercise on animal models of addiction to alcohol and other drugs of abuse. Such findings indicate that exercise can reduced both binge drinking and preference for alcohol in rodent models (Leasure and Nixon, 2010). Some small clinical studies have also shown the benefits of physical activity in combating alcohol and drug addiction in humans (Lynch et al., 2013; Stoutenberg et al., 2016). In addition to addiction, the benefits of physical training in psychological disorders ranging from depression to schizophrenia have been reported in patients undergoing an exercise regimen (Dauwan et al., 2016; McEwen et al., 2015). The fact that exercise has such wide reaching effects in a spectrum of brain disorders suggests that physical activity can tap into mechanisms of synaptic connectivity to restore homeostasis and that the mechanisms leading to these benefits may in fact be shared among distinct brain disorders.

\section{Clinical Studies Underway in Our Group}

While our ability to treat PD-like symptomology in our rodent models of dopamine depletion is noteworthy, the ultimate goal is to apply what we have learned to patients with PD and to elucidate the underlying mechanism. We (G. Petzinger, principal investigator) are currently engaged in a clinical trial with patients in the early stages of PD manifesting mild cognitive impairment (MCI). There are three groups including (i) social engagement, (ii) aerobic exercise, and (iii) skill-based exercise. The aerobic group will be involved in an exercise using a recombinant bicycle (a form of physical activity with minimal cognitive engagement), while the skill-based group performs an exercise program with high cognitive engagement and aerobic demand, all performed three times per week for an 8 week period (Voelcker-Rehage et al., 2010; 2011). The social engagement group serves as an important control since we do know that such interactions can influence neuroplasticity itself (Bassuk et al., 1999). The goal is to identify the differences in the activation of cognitive circuits, especially those from the prefrontal cortex, within these different groups. Subjects will be required to perform a motor task that utilizes both learning with a set-shifting component (Lee et al., 2015) while undergoing functional neuro-imaging. If our hypotheses are correct, we expect that the skill-based exercise group will show the greatest degree of prefrontal cortex activation and reduced cognitive impairment compared to the other two groups.

\section{Conclusions}

We are now beginning to better understand the parameters of physical activity and physiotherapy and how they may be applied not only to the aging brain, but also to those afflicted with neurological disorders such as cognitive impairment and PD. We are now in a position to convey to patients, their caregivers, and those who treat them including physical therapist and neurologists, that it is not necessarily the type of exercise that matters such as dance versus Tai Chi versus treadmill exercise, but rather the parameters within the physical activity itself. These parameters include task intensity (it must be high such that one may have to break a sweat), the task must be difficult to challenge the subject such that they are learning from the experience, the task must be specific to 
the motor circuits affected by disease or aging, and the task must be complex to engage the individual. Putting these parameters together indicates that physical activity and exercise, to maximize benefit for the patient, must be a learning modality with goal oriented targets and cognitive engagement. Cognition makes motor behaviors automatic. Deficits in cognition can create deficits in motor behavior, since the cognitive load and its demands on the brain can, in fact, be maladaptive and may reveal the major behavioral deficits when compensatory strategies are exhausted (Beeler et al., 2013).

Finally, not only is the elucidation of the underlying mechanisms of physical activity and exercise opening up new avenues to understanding neurological disorders, but it is also re-enforcing the importance of physical activity and motor learning through all stages of life from childhood, through adolescence, and into early and late adulthood. Along with diet, stress management, social engagement, physical activity is a critical component of a healthy lifestyle and our wellness throughout life. Judging from the complexity of motor learning and the integration of cognitive and motor circuits, one can easily appreciate that there never will be a pill to replace exercise.

\section{Acknowledgements}

The authors would like to thank those members of the laboratory who conduct the experiments and offer insightful contributions including Matthew Halliday, Daniel Stefanko, William Toy, Natalie Kintz, Lauren Hawthorne, Beth Fisher, Yumei Guo, Damaris Garcia, and John Walsh. We also thank those who support our studies including the Roberto Gonzalez Family Foundation, USC Collaboration Grant, Team Parkinson and those individual donors who make a difference.

\section{References}

Adlard PA, Cotman CW. Voluntary exercise protects against stress-induced decreases in brain-derived neurotrophic factor protein expression. Neuroscience, 2004; 124(4): 985-92

Altman J, Das GD. Autoradiographic and histological evidence of postnatal hippocampal neurogenesis in rats. J Comp Neurol, 1965; 124(3): 319-35

Archer T, Fredriksson A, Schutz E, Kostrzewa RM. Influence of physical exercise on neuroimmunological functioning and health: aging and stress. Neurotox Res, 2011; 20(1): 69-83

Bassuk SS, Glass TA, Berkman LF. Social disengagement and incident cognitive decline in communitydwelling elderly persons. Ann Intern Med, 1999; 131(3): 165-73

Bavelier D, Levi DM, Li RW, Dan Y, Hensch TK. Removing brakes on adult brain plasticity: from molecular to behavioral interventions. J Neurosci, 2010; 30(45): 14964-71

Beeler JA, Cao ZF, Kheirbek MA, Ding Y, Koranda J, Murakami M, Kang UJ, Zhuang X. Dopaminedependent motor learning: insight into levodopa's long-duration response. Ann Neurol, 2010; 67(5): 639-47

Beeler JA, Frank MJ, McDaid J, Alexander E, Turkson S, Bernandez MS, McGehee DS, Zhuang X. A role for dopamine-mediated learning in the pathophysiology and treatment of Parkinson's disease. Cell Rep, 2012; 2(6): 1747-61

Beeler JA, Petzinger G, Jakowec MW. The Enemy within: Propagation of Aberrant Corticostriatal Learning to Cortical Function in Parkinson's Disease. Front Neurol, 2013; 4134

Berchtold NC, Chinn G, Chou M, Kesslak JP, Cotman CW. Exercise primes a molecular memory for brainderived neurotrophic factor protein induction in the rat hippocampus. Neuroscience, 2005; 133(3): 85361

Berlucchi G. The origin of the term plasticity in the neurosciences: Ernesto Lugaro and chemical synaptic transmission. J Hist Neurosci, 2002; 11(3): 305-9

Berlucchi G, Buchtel HA. Neuronal plasticity: historical roots and evolution of meaning. Exp Brain Res, 2009; 192(3): 307-19 
Berti V, Polito C, Ramat S, Vanzi E, De Cristofaro MT, Pellicano G, Mungai F, Marini P, Formiconi AR, Sorbi $\mathrm{S}$, Pupi A. Brain metabolic correlates of dopaminergic degeneration in de novo idiopathic Parkinson's disease. Eur J Nucl Med Mol Imaging, 2010; 37(3): 537-44

Biglan KM, Zhang Y, Long JD, Geschwind M, Kang GA, Killoran A, Lu W, McCusker E, Mills JA, Raymond LA, Testa C, Wojcieszek J, Paulsen JS. Refining the diagnosis of Huntington disease: the PREDICT-HD study. Front Aging Neurosci, 2013; 512

Black JE, Isaacs KR, Anderson BJ, Alcantara AA, Greenough WT. Learning causes synaptogenesis, whereas motor activity causes angiogenesis, in cerebellar cortex of adult rats. Proc Natl Acad Sci U S A., 1990; 87(14): 5568-72

Bowling H, Bhattacharya A, Klann E, Chao MV. Deconstructing brain-derived neurotrophic factor actions in adult brain circuits to bridge an existing informational gap in neuro-cell biology. Neural regeneration research, 2016; 11(3): 363-7

Cameron HA, Glover LR. Adult neurogenesis: beyond learning and memory. Annu Rev Psychol, 2015; 665381

Caproni S, Muti M, Principi M, Ottaviano P, Frondizi D, Capocchi G, Floridi P, Rossi A, Calabresi P, Tambasco N. Complexity of motor sequences and cortical reorganization in Parkinson's disease: a functional MRI study. PLoS One, 2013; 8(6): e66834

Chen H, Zhang SM, Schwarzschild MA, Hernan MA, Ascherio A. Physical activity and the risk of Parkinson disease. Neurology, 2005; 64(4): 664-9

Cooper SJ. Donald O. Hebb's synapse and learning rule: a history and commentary. Neurosci Biobehav Rev., 2005; 28(8): 851-74

Correia SC, Moreira PI. Hypoxia-inducible factor 1: a new hope to counteract neurodegeneration? J Neurochem, 2010; 112(1): 1-12

Cotman CW, Berchtold NC, Christie LA. Exercise builds brain health: key roles of growth factor cascades and inflammation. Trends Neurosci, 2007; 30(9): 464-72

Dauwan M, Begemann MJ, Heringa SM, Sommer IE. Exercise Improves Clinical Symptoms, Quality of Life, Global Functioning, and Depression in Schizophrenia: A Systematic Review and Meta-analysis. Schizophr Bull, 2016; 42(3): 588-99

Dobrossy MD, Dunnett SB. Morphological and cellular changes within embryonic striatal grafts associated with enriched environment and involuntary exercise. Eur J Neurosci, 2006; 24(11): 3223-33

Dobrossy MD, Nikkhah G. Role of experience, training, and plasticity in the functional efficacy of striatal transplants. Prog Brain Res, 2012; 200303-28

Dyer AH, Vahdatpour C, Sanfeliu A, Tropea D. The role of Insulin-Like Growth Factor 1 (IGF-1) in brain development, maturation and neuroplasticity. Neuroscience, 2016; 32589-99

Eddy MC, Stansfield KJ, Green JT. Voluntary exercise improves performance of a discrimination task through effects on the striatal dopamine system. Learn Mem, 2014; 21(7): 334-7

El Aidy S, Stilling R, Dinan TG, Cryan JF. Microbiome to Brain: Unravelling the Multidirectional Axes of Communication. Adv Exp Med Biol, 2016; 874301-36

Eriksson PS, Perfilieva E, Bjork-Eriksson T, Alborn AM, Nordborg C, Peterson DA, Gage FH. Neurogenesis in the adult human hippocampus. Nat Med, 1998; 4(11): 1313-7

Evrensel A, Ceylan ME. The Gut-Brain Axis: The Missing Link in Depression. Clinical psychopharmacology and neuroscience: the official scientific journal of the Korean College of Neuropsychopharmacology, 2015; 13(3): $239-44$

Fisher BE, Li Q, Nacca A, Salem GJ, Song J, Yip J, Hui JS, Jakowec MW, Petzinger GM. Treadmill exercise elevates striatal dopamine D2 receptor binding potential in patients with early Parkinson's disease. Neuroreport, 2013; 24(10): 509-14

Fisher BE, Petzinger GM, Nixon K, Hogg E, Bremmer S, Meshul CK, Jakowec MW. Exercise-induced 
behavioral recovery and neuroplasticity in the 1-methyl-4-phenyl-1,2,3,6-tetrahydropyridine-lesioned mouse basal ganglia. J Neurosci Res, 2004; 77(3): 378-90

Foley TE, Fleshner M. Neuroplasticity of Dopamine Circuits After Exercise: Implications for Central Fatigue. Neuromolecular Med, 2008; 10(2): 67-80

Garcia PC, Real CC, Ferreira AF, Alouche SR, Britto LR, Pires RS. Different protocols of physical exercise produce different effects on synaptic and structural proteins in motor areas of the rat brain. Brain Res, 2012; 145636-48

Gilliam PE, Spirduso WW, Martin TP, Walters TJ, Wilcox RE, Farrar RP. The effects of exercise training on [3H]-spiperone binding in rat striatum. Pharmacol Biochem Behav., 1984; 20(6): 863-7

Hamaide J, De Groof G, Van der Linden A. Neuroplasticity and MRI: A perfect match. Neuroimage, 2016; 13: 113-28

Kang SS, Jeraldo PR, Kurti A, Miller ME, Cook MD, Whitlock K, Goldenfeld N, Woods JA, White BA, Chia $\mathrm{N}$, Fryer JD. Diet and exercise orthogonally alter the gut microbiome and reveal independent associations with anxiety and cognition. Mol Neurodegener, 2014; 9(1): 36

Kannarkat GT, Boss JM, Tansey MG. The role of innate and adaptive immunity in Parkinson's disease. Journal of Parkinson's disease, 2013; 3(4): 493-514

Kintz N, Petzinger GM, Akopian G, Ptasnik S, Williams C, Jakowec MW, Walsh JP. Exercise modifies alphaamino-3-hydroxy-5-methyl-4-isoxazolepropionic acid receptor expression in striatopallidal neurons in the 1-methyl-4-phenyl-1,2,3,6-tetrahydropyridine-lesioned mouse. J Neurosci Res, 2013; 91(11): 1492507

Kornack DR, Rakic P. Continuation of neurogenesis in the hippocampus of the adult macaque monkey. Proc Natl Acad Sci U S A, 1999; 96(10): 5768-73

Leasure JL, Nixon K. Exercise neuroprotection in a rat model of binge alcohol consumption. Alcohol Clin Exp Res, 2010; 34(3): 404-14

Lee YY, Winstein CJ, Gordon J, Petzinger GM, Zelinski EM, Fisher BE. Context-Dependent Learning in People With Parkinson's Disease. J Mot Behav, 2015; 1-9

Lynch WJ, Peterson AB, Sanchez V, Abel J, Smith MA. Exercise as a novel treatment for drug addiction: A neurobiological and stage-dependent hypothesis. Neurosci Biobehav Rev, 2013; 37(8): 1622-44

MacRae PG, Spirduso WW, Walters TJ, Farrar RP, Wilcox RE. Endurance training effects on striatal D2 dopamine receptor binding and striatal dopamine metabolites in presenescent older rats. Psychopharmacology (Berl), 1987; 92(2): 236-40

Mayer EA, Tillisch K, Gupta A. Gut/brain axis and the microbiota. J Clin Invest, 2015; 125(3): 926-38

McEwen SC, Hardy A, Ellingson BM, Jarrahi B, Sandhu N, Subotnik KL, Ventura J, Nuechterlein KH. Prefrontal and Hippocampal Brain Volume Deficits: Role of Low Physical Activity on Brain Plasticity in First-Episode Schizophrenia Patients. J Int Neuropsychol Soc, 2015; 21(10): 868-79

Meissner WG. When does Parkinson's disease begin? From prodromal disease to motor signs. Rev Neurol (Paris), 2012; 168(11): 809-14

Menalled LB, Sison JD, Dragatsis I, Zeitlin S, Chesselet MF. Time course of early motor and neuropathological anomalies in a knock-in mouse model of Huntington's disease with 140 CAG repeats. J Comp Neurol, 2003; 465(1): 11-26

Mirelman A, Maidan I, Deutsch JE. Virtual reality and motor imagery: Promising tools for assessment and therapy in Parkinson's disease. Mov Disord, 2013; 28(11): 1597-608

Mirelman A, Maidan I, Herman T, Deutsch JE, Giladi N, Hausdorff JM. Virtual reality for gait training: can it induce motor learning to enhance complex walking and reduce fall risk in patients with Parkinson's disease? J Gerontol A Biol Sci Med Sci, 2011; 66(2): 234-40

Nemani K, Hosseini Ghomi R, McCormick B, Fan X. Schizophrenia and the gut-brain axis. Prog Neuropsychopharmacol Biol Psychiatry, 2014; 56c155-60 
Petzinger GM, Fisher BE, Akopian G, Holschneider DP, Wood R, Walsh JP, Lund B, Meshul C, Vuckovic M, Jakowec MW. The role of exercise in facilitating basal ganglia function in Parkinson's disease. Neurodegener Dis Manag, 2011; 1(2): 157-70

Petzinger GM, Holschneider DP, Fisher BE, McEwen S, Kintz N, Halliday M, Toy W, Walsh JW, Beeler J, Jakowec MW. The Effects of Exercise on Dopamine Neurotransmission in Parkinson's Disease: Targeting Neuroplasticity to Modulate Basal Ganglia Circuitry. Brain plasticity, 2015; 1(1): 29-39

Petzinger GM, Walsh JP, Akopian G, Hogg E, Abernathy A, Arevalo P, Turnquist P, Vuckovic M, Fisher BE, Togasaki DM, Jakowec MW. Effects of treadmill exercise on dopaminergic transmission in the 1methyl-4-phenyl-1,2,3,6-tetrahydropyridine-lesioned mouse model of basal ganglia injury. J Neurosci, 2007; 27(20): 5291-300

Polito C, Berti V, Ramat S, Vanzi E, De Cristofaro MT, Pellicano G, Mungai F, Marini P, Formiconi AR, Sorbi S, Pupi A. Interaction of caudate dopamine depletion and brain metabolic changes with cognitive dysfunction in early Parkinson's disease. Neurobiol Aging, 2012; 33(1): 206 e29-39

Ray K. Gut microbiota: Tackling the effects of diet and exercise on the gut microbiota. Nature reviews. Gastroenterology \& hepatology, 2014; 12(6): 311

Rosenzweig MR, Krech D, Bennett EL, Diamond MC. Effects of environmental complexity and training on brain chemistry and anatomy: a replication and extension. J Comp Physiol Psychol, 1962; 55429-37

Schafer DP, Lehrman EK, Kautzman AG, Koyama R, Mardinly AR, Yamasaki R, Ransohoff RM, Greenberg ME, Barres BA, Stevens B. Microglia sculpt postnatal neural circuits in an activity and complementdependent manner. Neuron, 2012; 74(4): 691-705

Scheperjans F, Aho V, Pereira PA, Koskinen K, Paulin L, Pekkonen E, Haapaniemi E, Kaakkola S, EerolaRautio J, Pohja M, Kinnunen E, Murros K, Auvinen P. Gut microbiota are related to Parkinson's disease and clinical phenotype. Mov Disord, 2015; 30(3): 350-8

Selkoe DJ. Preventing Alzheimer's disease. Science, 2012; 337(6101): 1488-92

Shatz CJ. The developing brain. Sci Am, 1992; 267(3): 60-7

Spurzheim JG. The Physiognomical System of Drs. Gall and Spurzheim. Baldwin, Cradock, and Joy, Edinburgh, Scotland; 1815

Stahnisch FW. Making the brain plastic: early neuroanatomical staining techniques and the pursuit of structural plasticity, 1910-1970. J Hist Neurosci, 2003; 12(4): 413-35

Stoutenberg M, Rethorst CD, Lawson O, Read JP. Exercise training - A beneficial intervention in the treatment of alcohol use disorders? Drug Alcohol Depend, 2016; 1602-11

Svensson M, Lexell J, Deierborg T. Effects of Physical Exercise on Neuroinflammation, Neuroplasticity, Neurodegeneration, and Behavior: What We Can Learn From Animal Models in Clinical Settings. Neurorehabil Neural Repair, 2015; 29(6): 577-89

Tabrizi SJ, Scahill RI, Owen G, Durr A, Leavitt BR, Roos RA, Borowsky B, Landwehrmeyer B, Frost C, Johnson H, Craufurd D, Reilmann R, Stout JC, Langbehn DR, Investigators T-H. Predictors of phenotypic progression and disease onset in premanifest and early-stage Huntington's disease in the TRACK-HD study: analysis of 36-month observational data. Lancet Neurol, 2013; 12(7): 637-49

Tang Y, Le W. Differential Roles of M1 and M2 Microglia in Neurodegenerative Diseases. Mol Neurobiol, 2016; 53(2): 1181-94

Tanzi E. A Textbook of Medical Diseases. Rebman Company, New York, New York; 1909

Thacker EL, Chen H, Patel AV, McCullough ML, Calle EE, Thun MJ, Schwarzschild MA, Ascherio A. Recreational physical activity and risk of Parkinson's disease. Mov Disord, 2008; 23(1): 69-74

Tolosa E, Pont-Sunyer C. Progress in defining the premotor phase of Parkinson's disease. J Neurol Sci, 2011; 310(1-2): 4-8

van Dellen A, Grote HE, Hannan AJ. Gene-environment interactions, neuronal dysfunction and pathological plasticity in Huntington's disease. Clin Exp Pharmacol Physiol, 2005; 32(12): 1007-19 
van Praag H, Christie BR, Sejnowski TJ, Gage FH. Running enhances neurogenesis, learning, and long-term potentiation in mice. Proc Natl Acad Sci U S A., 1999; 96(23): 13427-31

van Praag H, Shubert T, Zhao C, Gage FH. Exercise enhances learning and hippocampal neurogenesis in aged mice. J Neurosci, 2005; 25(38): 8680-5

VanLeeuwen JE, Petzinger GM, Walsh JP, Akopian GK, Vuckovic M, Jakowec MW. Altered AMPA receptor expression with treadmill exercise in the 1-methyl-4-phenyl-1,2,3,6-tetrahydropyridine-lesioned mouse model of basal ganglia injury. J Neurosci Res, 2010; 88(3): 650-68

Voelcker-Rehage C, Godde B, Staudinger UM. Physical and motor fitness are both related to cognition in old age. Eur J Neurosci, 2010; 31(1): 167-76

Voelcker-Rehage C, Godde B, Staudinger UM. Cardiovascular and coordination training differentially improve cognitive performance and neural processing in older adults. Front Hum Neurosci, 2011; 526

Vuckovic MG, Li Q, Fisher B, Nacca A, Leahy RM, Walsh JP, Mukherjee J, Williams C, Jakowec MW, Petzinger GM. Exercise elevates dopamine D2 receptor in a mouse model of Parkinson's disease: in vivo imaging with [(1)F]fallypride. Mov Disord, 2010; 25(16): 2777-84

Wang Z, Guo Y, Myers KG, Heintz R, Peng YH, Maarek JM, Holschneider DP. Exercise alters resting-state functional connectivity of motor circuits in parkinsonian rats. Neurobiol Aging, 2015; 36(1): 536-44

Wang Z, Myers KG, Guo Y, Ocampo MA, Pang RD, Jakowec MW, Holschneider DP. Functional reorganization of motor and limbic circuits after exercise training in a rat model of bilateral parkinsonism. PLoS One, 2013; 8(11): e80058

Yang J, Sadler TR, Givrad TK, Maarek JM, Holschneider DP. Changes in brain functional activation during resting and locomotor states after unilateral nigrostriatal damage in rats. Neuroimage, 2007; 36(3): 75573

Yim AJ, Andersen ML, Soeiro AC, Tufik S, Oliveira MG. Acute systemic blockade of D2 receptors does not accelerate the extinction of cocaine-associated place preference. Brain Res, 2009: 1304122-8

\section{Corresponding author:}

\section{Michael Jakowec}

Department of Neurology, University of Southern California, 1333 San Pablo St., MCA-241, Los Angeles, California, USA, 90033

E-mail: mjakowec@surgery.usc.edu 